\title{
Erratum: Towards graded-index magnonics: Steering spin waves in magnonic networks [Phys. Rev. B 92, 020408(R) (2015)]
}

\author{
C. S. Davies, A. Francis, A. V. Sadovnikov, S. V. Chertopalov, M. T. Bryan, S. V. Grishin, \\ D. A. Allwood, Y. P. Sharaevskii, S. A. Nikitov, and V. V. Kruglyak \\ (Received 13 December 2016; published 3 January 2017)
}

DOI: 10.1103/PhysRevB.95.019901

The horizontal axis of the color map from Fig. 3(a) was mistakenly flipped. The correct version of Fig. 3 is given below (the caption is the same).
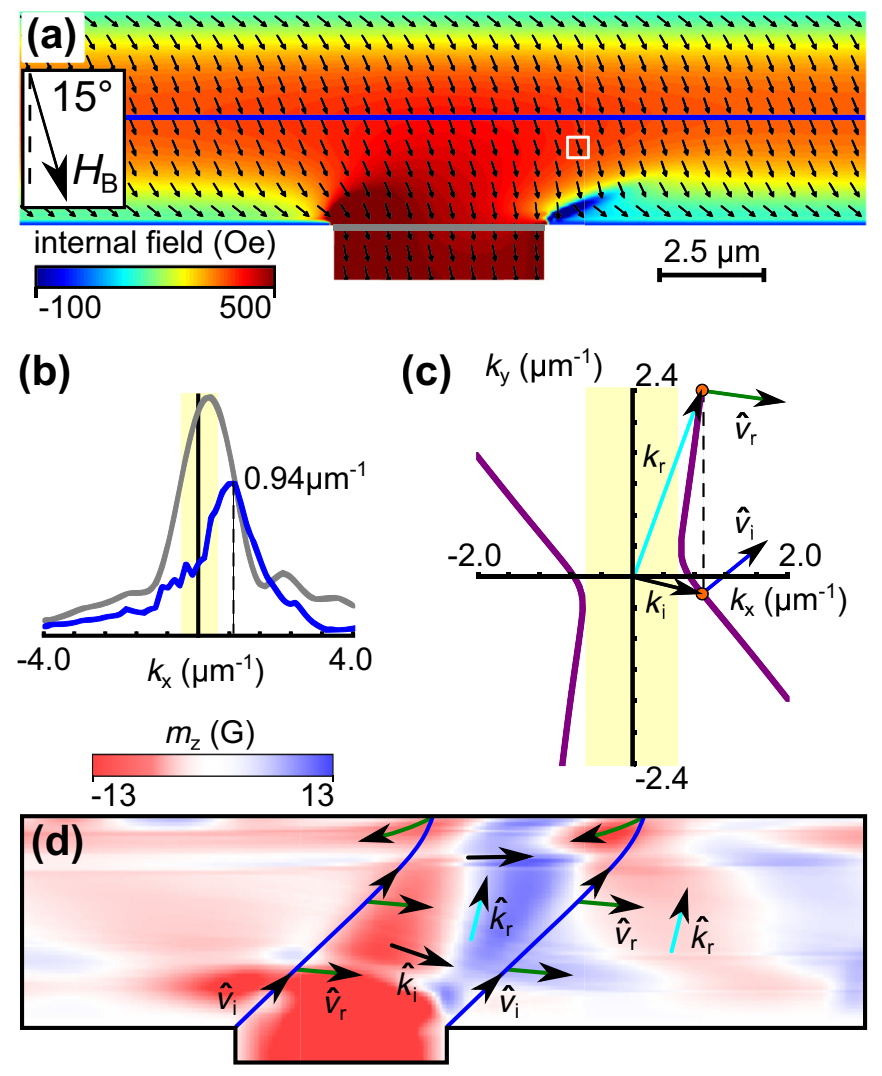

FIG. 3. Spin waves in an asymmetrically magnetized permalloy $T$ junction. (a) The calculated distributions of the static magnetization (arrows) and the projection of the internal magnetic field onto the magnetization (color scale) are shown for the magnetic field of $H_{\mathrm{B}}=500 \mathrm{Oe}$ applied at $15^{\circ}$ to the vertical symmetry axis. Each arrow represents the average of $5 \times 5$ mesh cells. (b) $k_{x}$ spectra of the dynamic magnetization distributions across the leg [gray line in (a)] and along the arms [blue line in (a), amplified $\times 5$ ) of the $T$ junction excited at $7.52 \mathrm{GHz}$ are shown by the gray and blue curves, respectively. The $k_{x}$ value of $0.94 \mu \mathrm{m}^{-1}$ indicated by a dashed line corresponds to maximum spin-wave Fourier amplitude. (c) A construction illustrating the extraction of the group velocities of the incident (index " $i$ ") and reflected (index " $r$ ") beams is shown for the white boxed pixel in (a). For the specific $k_{x}$ value of $0.94 \mu \mathrm{m}^{-1}$ [which is indicated by the vertical dashed lines here and in panel (b)], the group velocities are perpendicular to the characteristic isofrequency curves (purple). The $k_{y}$ value is given by the crossing of the dashed line and the isofrequency curve. Here and in panel (b), the region shaded in yellow has the same size and represents the range of forbidden $k_{x}$ values as calculated for the white boxed pixel and for pixels on the gray line in (a), respectively. (d) The extracted directional unit vectors of the group-velocities $\hat{v}$ and wave-vectors $\hat{k}$ are shown for $k_{x}$ values shown by the vertical dashed lines in (b) and (c).

A separate minor error was in the code used to perform calculations for Fig. S5 in the Supplemental Material [1]. The correct version of Fig. S5 is given below (the caption is the same).

The same error also affected Fig. S6(a) in the Supplemental Material [1]. The correct version of Fig. S6 is given below (the caption is the same).

None of the plotting mistakes affected any subsequent calculations in which correct data was used or conclusions. 

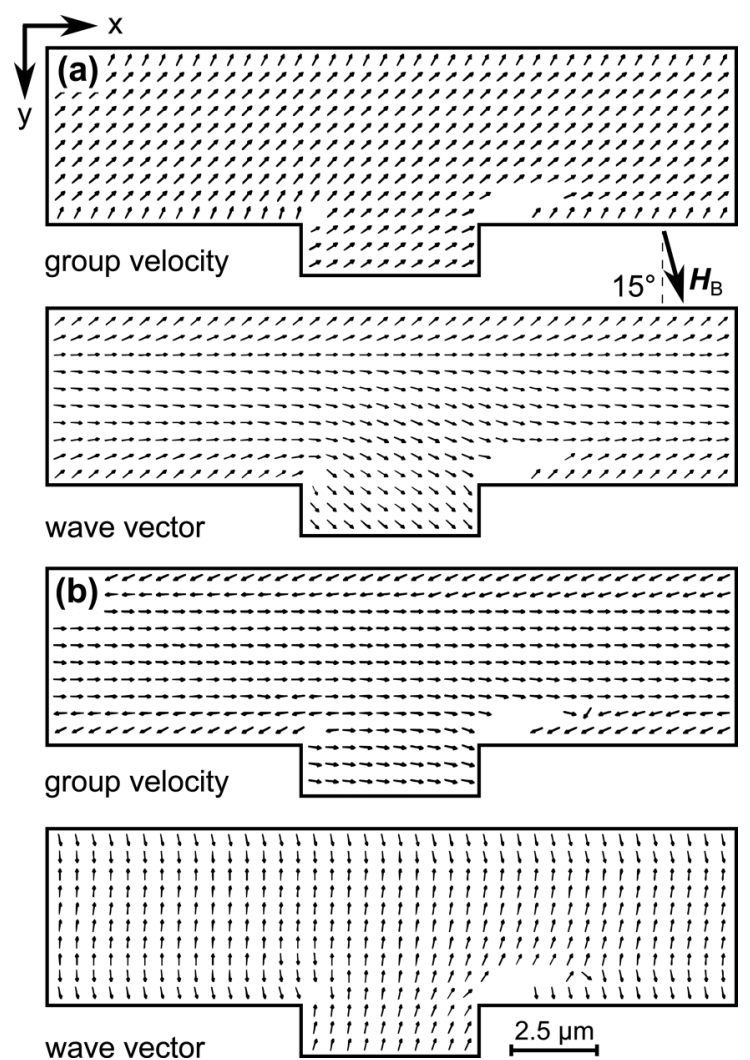

FIG. S5. The vector maps of the spatial variation of the group velocity (top panel) and the wave vector (bottom panel) in the permalloy $T$ junction are shown for the (a) incident and (b) scattered spin waves with the horizontal projection of the wave vector of $k_{x}=0.94 \mu \mathrm{m}^{-1}$. Each unit vector represents an average over $5 \times 5$ computational cells.
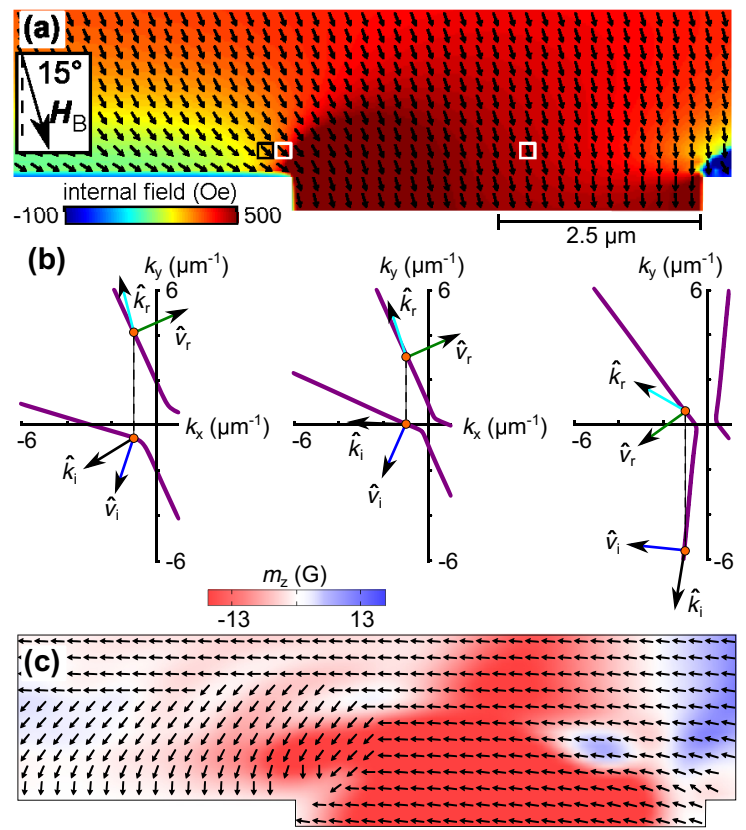

FIG. S6. (a) The static magnetic state of the permalloy $T$ junction is shown for the bias magnetic field applied at $+15^{\circ}$ to the symmetry axis. The image is a zoomed copy of that presented in Fig. 3(a) with a focus on the left side of the junction-arms interface. The arrows represent the average direction of the magnetization for $2 \times 2$ simulation cells. The color scale represents the projection of the internal field on the magnetization direction. (b) Three characteristic sets of isofrequency curves are shown for the boxed pixels in panel (a) in respective order. Indices $i$ and $r$ correspond to the unit vectors of the wave-vectors $\hat{k}$ and group-velocity $\hat{v}$ the incident and reflected waves, respectively. (c) The spatial distribution of the group-velocity unit vectors is shown for $k_{x}=-1 \mu \mathrm{m}^{-1}$. 
[1] See Supplemental Material at http://link.aps.org/supplemental/10.1103/PhysRevB.95.019901 for additional details of the TRSKM measurements and micromagnetic simulations and movies of the spin-wave propagation associated with the presented images. 